\title{
Third nerve palsy due to posterior communicating artery aneurysm: the importance of early surgery
}

\author{
MICHAEL FEELY, SAMIR KAPOOR \\ From the Department of Neurosurgery, Cork Regional Hospital, Wilton, Cork, Ireland
}

SUMMARY The value of early, direct aneurysm clipping in restoring third-nerve function after palsy associated with a posterior communicating artery aneurysm is assessed.

The sudden onset of a painful third nerve palsy is readily recognised. That it is frequently due to a carotid artery aneurysm at the take-off of the posterior communicating artery (PCA) is widely known. Treatment has principally focused on prevention of a full blown subarachnoid haemorrhage. Less attention has been given to recovery of third nerve function, yet even a minor degree of residual extra-occular muscle weakness may cause diplopia leading to effective loss of vision in one eye. To analyse factors that might influence recovery of third nerve function we retrospectively studied the records of all patients who had third nerve palsy due to PCA aneurysm at our department between November 1981 and January 1985.

\section{Patients \\ There were 13 patients, $20 \%$ of PCA aneurysms operated on during this period. Ten were females and three males; eight were right sided and five left sided aneurysms. Ages ranged from 26 to 71 years (average 51 years). Twelve patients presented within one week of the onset of palsy and one patient presented one month after the onset of palsy. Two patients had partial palsy and 11 had complete palsy. Ten patients had pain in the orbital region, beginning from 2 days to 1 month (average 12 days) before the onset of palsy. Two patients had a simultaneous onset of third nerve palsy and generalised subarachnoid haemorrhage. In these the records do not specify pain in the orbital region separate from generalised headache. Palsy was painless in one case.}

Address for reprint requests: Mr M Feely, Department of Neurosurgery, Cork Regional Hospital, Wilton, Cork, Ireland.

Received 5 December 1986 and in revised form 7 February 1987. Accepted 11 February 1987
Five patients had generalised subarachnoid haemorrhage, two before, two simultaneous with, and one after the onset of palsy.

\section{Results}

All patients had angiography and surgery within 3 days of admission. All patients were followed until recovery or until the palsy had remained static for at least 6 months. The two patients who had a partial palsy made an early and complete recovery.

Of the 11 patients who presented with complete palsy, nine made a full functional recovery. The one patient who presented with a 1 month history of palsy had no improvement, and the complete palsy was unchanged 6 months after surgery. The other patient made a partial recovery, but is bothered by persisting diplopia and photophobia and keeps the affected eye patched.

Overall 9 of the 10 patients who had a complete palsy and had surgery within 8 days of the onset had full functional recovery. Two of these have some residual dilation of the pupil, but have normal vision.

\section{Discussion}

Of all third nerve palsies $13.8 \%$ have been reported as due to intracranial aneurysm. ${ }^{1}$ The first major study on aneurysmal third nerve palsy is that of Jefferson ${ }^{2}$ in 1946. He pointed out that angiography is the only way of making the diagnosis with certainty. He recommended carotid ligation principally to prevent recurrent haemorrhage. He gives no information on the recovery of third nerve palsy. Botterell et al published the first major study on recovery of third nerve function. ${ }^{3}$ Seven of their 18 patients with complete 
palsy were operated on within 10 days of the onset. There was one death and three of the remaining six patients made a complete recovery. None of the 11 patients operated on for direct clipping after 10 days had a complete recovery.

Perneczky and $\mathrm{Czech}^{4}$ recently reviewed the factors that improve the prognosis for recovery. They outlined three: degree of paralysis, timing of surgery and surgical reduction of the aneurysm size. All three patients in their series with incomplete palsy made a full recovery. Five of nine patients operated on within 14 days made a full recovery. Only one of 11 patients who had surgery after 14 days made a full recovery. They did not advance any particular evidence as to why decompressing the nerve by reducing the aneurysm size helped improve the outcome.

In our series partial palsy at the time of surgery was followed by an early and rapid recovery. Nine of 10 patients who had complete palsy and had surgery within 8 days of the onset made a full recovery.

Hyland and Barnett ${ }^{5}$ have shown that the commonest cause of complete palsy is haemorrhage into the substance of the nerve. What happens to the nerve during the second week after the onset of palsy and renders it incapable of regeneration, is as yet unknown. The fact that early surgical clipping without further decompression of the nerve, in our and other hands, allows the nerve to recover suggests that there may be a high incidence of further minor haemorrhage into the nerve during the second week after the onset of palsy and that this leads to the permanent paralysis.
From this small series and review of other reports, some conclusions can be drawn. Firstly, direct clipping of the aneurysm leads to an excellent prospect of recovery in cases of incomplete palsy or in cases of complete palsy where surgery is performed within 8 days of the onset. Secondly, from a review of other studies, as outlined above ${ }^{34}$ delaying surgery beyond the period of 10 to 14 days after the onset of complete palsy would make a satisfactory recovery most unlikely.

We thank Mr TF Buckley, FRCS, for permission to report his cases.

\section{References}

1 Rush JA, Younge BR. Paralysis of cranial nerves III, IV, and VI. Cause and prognosis in 1,000 cases. Arch Oph- $\vec{\circ}$ thalmol 1981;99:76-9.

2 Jefferson G. Isolated Oculomotor palsy caused by intra- $\bar{\omega}$ cranial aneurysm. Proc $R$ Soc Med 1947;XL:419-32.

3 Botterell EH, Lloyd LA, Hoffman HJ. Oculomotor palsy due to supraclinoid internal carotid artery berry aneurysm. A long-term study of the results of surgical of treatments on the recovery of third-nerve function Am J Ophthalmol 1962;54:609-16.

4 Perneczky A, Czech T. Prognosis of oculomotor palso 앙 following subarachnoid haemorrhage due to aneurysms of the posterior communicating arters Zentralbl Neurochirurgie 1984;45:189-95.

5 Hyland HH, Barnett HJM. The Pathogenesis of crania nerve palsies associated with intracranial aneurysm 8 Proc R Soc Med 1954;47:141-6. 